\title{
Improving the Reproducibility of P3HT:PCBM Solar Cells by Controlling the PCBM/ Cathode Interface
}

\author{
Bertrand Tremolet de Villers, Christopher J. Tassone, Sarah H. Tolbert,* and \\ Benjamin J. Schwartz* \\ Department of Chemistry and Biochemistry, University of California, Los Angeles, Los Angeles, California 90095-1569
}

Received: August 25, 2009; Revised Manuscript Received: September 17, 2009

\begin{abstract}
Plastic photovoltaic devices offer a real potential for making solar energy economically viable. Unfortunately, bulk heterojunction (BHJ) solar cells fabricated from blends of the commonly used materials poly(3hexylthiophene), $\mathrm{P} 3 \mathrm{HT}$, and phenyl- $\mathrm{C}_{61}$-butyric acid methyl ester, $\mathrm{PCBM}$, sometimes exhibit low efficiencies even when the procedures followed often produce solar cells with efficiencies exceeding 5\%. In this Letter, we show that this irreproducibility is caused by subtleties in the film processing conditions that ultimately lead to poor electron extraction from the devices. For low-performing devices, photogeneration and charge extraction with a linearly increasing voltage ramp (photo-CELIV) measurements show an order-of-magnitude difference in the effective mobilities of the electrons and holes. Atomic force microscopy (AFM) experiments reveal that the top surface of these low-performing devices is nearly pure P3HT. We argue that small variations in the solvent evaporation kinetics during spin-coating of the BHJ active layer, which are difficult to control, cause PCBM to segregate toward the bottom of the P3HT film to different extents, explaining why electron extraction from the PCBM component of the BHJ is so difficult in poorly performing devices. Finally, we show that electron extraction can be greatly improved by spin-coating a thin PCBM layer on top of the BHJ before deposition of the cathode, allowing the reproducible fabrication of high-efficiency polymer solar cells.
\end{abstract}

Organic photovoltaics based on bulk-heterojunction (BHJ) composites of conjugated polymers and fullerenes have shown rapid improvement in the past few years, ${ }^{1,2}$ with power conversion efficiencies recently surpassing $6 \% .^{3}$ Although facile, solution-phase fabrication is one of the greatest advantages this class of solar cells has over its inorganic-based counterparts. The behavior of polymer/fullerene devices is sensitive to small variations in processing conditions; ${ }^{4,5}$ for example, small changes in material blend ratios, ${ }^{6}$ single-percent variations in the composition of the solvent used for spin-coating, ${ }^{7,8}$ and changes in postfabrication treatments such as the time and/or temperature of thermal annealing ${ }^{9}$ all can dramatically affect device performance. Perhaps even more troublesome, there is not always good reproducibility when different groups use the same processing recipe for producing polymer/fullerene thin-film photovoltaic devices, indicating that there are still processing parameters that we have not yet either correctly identified or properly learned to control in order to consistently optimize device performance.

A prime example of this lack of reproducibility can be seen in BHJ devices fabricated from blends of the commonly used materials regioregular poly(3-hexylthiophene), $\mathrm{P} 3 \mathrm{HT}$, and phenyl- $\mathrm{C}_{61}$-butyric acid methyl ester, PCBM. BHJ solar cells fabricated from these materials can have power conversion efficiencies (PCEs) exceeding 5\%,9,10 but sometimes, cells fabricated with nominally identical processing conditions can exhibit efficiencies of less than $1 \%$. The low-performing devices often have an S-shaped current versus applied voltage $(J-V)$

* To whom correspondence should be addressed. E-mail: schwartz@ chem.ucla.edu (B.J.S.); tolbert@chem.ucla.edu (S.H.T.). behavior under illumination that results in exceedingly low fill factors $(\sim 10-25 \%$.) Figure 1 shows $J-V$ curves observed in our laboratory for P3HT:PCBM-based BHJ solar cells fabricated using nominally identical processing conditions. The solid curve shows "typical" $J-V$ characteristics with a high fill factor and good PCE. The dashed curve, on the other hand, shows a device with a similar short-circuit current $\left(J_{\mathrm{sc}}\right)$ and open-ciruit voltage $\left(V_{\text {oc }}\right)$ but a fill factor that is significantly reduced by the presence of the "S curve". Anecdotal evidence suggests that few groups, if any, can reproducibly make $\geq 5 \%$-efficient devices every time they try and that nearly every group in the field fabricates devices that show the $\mathrm{S}$ curve from time to time. The sporadic nature of the $\mathrm{S}$ curve, however, has prevented widespread discussion about it in the literature; we are aware of only a few papers that have investigated the origins of the $S$ curve in polymer:fullerene BHJ solar cells. ${ }^{11-15}$

In this Letter, we identify the cause of the $S$ curve in P3HT:PCBM BHJ devices and provide a method to reproducibly eliminate the low fill factor through simple changes in the device architecture. Using the transient current technique known as photogeneration and charge extraction with a linearly increasing voltage ramp (photo-CELIV), ${ }^{16}$ we first show that the $S$ curve results from poor contact between the PCBM component of the $\mathrm{BHJ}$ and the cathode, which hinders electron extraction and leads to an imbalance in the rates at which holes and electrons are extracted from the active layer. We then argue based on atomic force microscopy (AFM) experiments that the poor extraction of charge at the cathode interface is due to undesirable vertical phase segregation in the $\mathrm{BHJ}$ blend, the result of solvent evaporation kinetics that can cause PCBM to segregate to the 


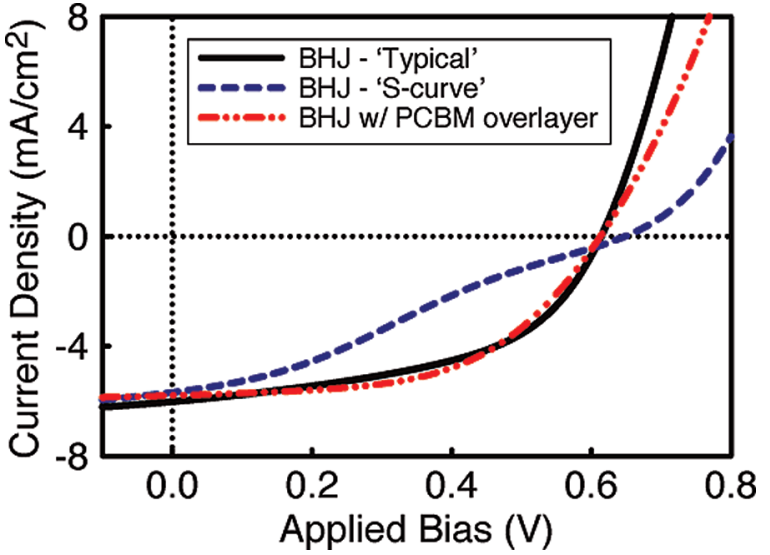

Figure 1. Current - voltage characteristics under AM-1.5 illumination for P3HT:PCBM BHJ solar cells. The solid curve shows the performance of a "typical" ITO/PEDOT:PSS/P3HT:PCBM/Al BHJ solar cell with a $\sim 140 \mathrm{~nm}$ thick active layer, which exhibits a good fill factor $(50 \%)$ and reasonable power conversion efficiency (PCE). The dashed curve shows the $J-V$ behavior of a device fabricated under nominally identical conditions as that for the solid curve but which has a low fill factor $(25 \%)$ and PCE due to the presence of the "S curve"; even when the $\mathrm{S}$ curve is not present, the $J-V$ curves of cells made on different days using the same nominal processing conditions are often different. The dot-dashed curve shows the $J-V$ characteristics of a BHJ solar cell fabricated at the exact same time as the $S$ curve device whose $J-V$ behavior is shown by the dashed curve but which had an additional PCBM overlayer spun on top of the BHJ film, restoring the good fill factor $(54 \%)$ and PCE. In addition to the improvement in performance, we have never fabricated a BHJ device with a PCBM overlayer that shows the S curve, and we find that devices with PCBM overlayers give much more reproducible $J-V$ behavior.

bottom of the film ${ }^{17,18}$ to different extents depending on subtle variations in the BHJ film processing conditions. Finally, we show that the $\mathrm{S}$ curve can be reproducibly eliminated by spincoating a thin additional layer of PCBM on top of the BHJ, which improves the extraction of electrons from the device by "repairing" the poor contact at the PCBM/cathode interface.

The solar cells that we describe below were fabricated by first spin-coating a $\leq 50 \mathrm{~nm}$ thick layer of poly(3,4-ethylenedioxythiophene):poly(styrenesulfonate) (PEDOT:PSS, Clevios $\mathrm{P}$ VP A14083) onto indium tin oxide (ITO)-coated glass substrates (TFD, Inc.). The active layers were then deposited by spin-coating (at $600 \mathrm{rpm}$ for $5 \mathrm{~min}$ ) an $o$-dichorlorbenzene (ODCB) solution of P3HT (2\% w/v, Rieke Metals)) and PCBM (Nano-C) mixed in a 1:0.8 weight ratio to produce a $\sim 140 \mathrm{~nm}$ thick BHJ film. We then thermally evaporated $70 \mathrm{~nm}$ thick aluminum (99.99\%, Kurt J. Lesker) cathodes onto the structures through a shadow mask, producing devices with an active area of $4 \mathrm{~mm}^{2}$. The devices discussed in this paper were thermally annealed at $150{ }^{\circ} \mathrm{C}$ for $20 \mathrm{~min}$ while covered with a shallow glass Petri dish. Additional details of our methods for substrate preparation, device fabrication, and device characterization under AM-1.5 illumination are described in the Supporting Information. Atomic force microscopy (AFM) was carried out using a Nanoscope V Dimension 5000 (Veeco Digital Instruments) in ambient conditions. Antimony $n$-doped silicon cantilevers (TESPW, Veeco Probes) with spring constants of $42 \mathrm{~N} / \mathrm{m}$, first longitudinal resonance frequencies between $230-410 \mathrm{kHz}$, and nominal tip radii of $8 \mathrm{~nm}$ were employed in tapping mode. Simultaneous height and phase images were acquired and reproduced across multiple samples.

To understand the nature of the charge extraction and carrier mobility in P3HT:PCBM solar cells with the $\mathrm{S}$ curve, we used photo-CELIV, ${ }^{16,19}$ a technique that has been previously applied to examine the transport and recombination of photogenerated carriers in polymer:fullerene thin-film BHJ solar cells..$^{20-23} \mathrm{In}$ our photo-CELIV measurements, we partly compensated for the built-in potential that exists within the device by first applying a small, constant offset bias $(+0.07$ to $+0.18 \mathrm{~V}$, depending on the sample) to help prevent photogenerated charge carriers from migrating toward their respective electrodes. We then illuminated the sample with a $\sim 7 \mathrm{~ns}$ pulse of light at 500 $\mathrm{nm}$ from a $10 \mathrm{~Hz} \mathrm{~N}_{2}$-pumped dye laser to create charge carriers within the polymer:fullerene blend film. After an adjustable time delay, $t_{\mathrm{del}}$, controlled by a digital delay generator (SRS DG535), we applied a reverse-bias voltage ramp with a slope of $4 \mathrm{~V} / 100$ $\mu$ s created with an arbitrary function generator (SRS DS345) to extract any carriers that had not recombined. We then monitored the resulting current, $j$, as a function of time using the $50 \Omega$ input of a digital oscilloscope (Tektronix DPO3014). The top panel of Figure 2a depicts the time sequence of the applied light pulse and voltages. The bottom panel of Figure 2a shows a typical corresponding measured current transient. The shaded part of the current transient, with amplitude labeled $\Delta j$, represents the extraction of the photogenerated charge carriers. This signal appears on top of the rectangular capacitive current, $j_{0}$, which has a typical magnitude of $0.3 \mathrm{~mA} / \mathrm{cm}^{2}$ for our devices; in the data presented below, we have subtracted the capacitive current, measured when the light pulse is blocked, to better focus on the dynamics of the photogenerated carriers extracted by the voltage ramp.

Figure $2 \mathrm{~b}$ shows photo-CELIV current transients, collected at different values of $t_{\mathrm{del}}$, for a P3HT:PCBM BHJ solar cell that has an S-shaped $J-V$ curve under AM-1.5 illumination, similar to that shown by the dashed curve in Figure 1. The current transients clearly show two distinct extraction peaks at $t_{\max } \approx$ 2 and $12 \mu \mathrm{s}$, a signature that, to the best of our knowledge, has not been previously reported for polymer-based photovoltaic devices. The fact that two peaks are observed in the current transient signifies that the extraction of holes at the anode/BHJ interface occurs at a different rate than the extraction of electrons at the $\mathrm{BHJ} /$ cathode interface. We can get a rough estimate of the mobilities of the carriers responsible for the two peaks in the current transient from

$$
\mu=2 d^{2}\left[3 A t_{\max }^{2}\left(1+0.36 \Delta j / j_{0}\right)\right]^{-1}
$$

where $\mu$ is the charge carrier mobility, $d$ is the thickness of the active layer, $A$ is the slope of the extraction volage ramp, $t_{\max }$ is the time at which the extracted reaches its peak, $\Delta j$ is the current extraction peak height, and $j_{0}$ is the value of the dark, capacitive current, as shown in Figure $2 \mathrm{a}^{24}$ For the data in Figure $2 \mathrm{~b}$, we find that $\mu_{\text {fast }}=2 \times 10^{-4} \mathrm{~cm}^{2} \mathrm{~V}^{-1} \mathrm{~s}^{-1}$ and $\mu_{\text {slow }}$ $=1 \times 10^{-5} \mathrm{~cm}^{2} \mathrm{~V}^{-1} \mathrm{~s}^{-1}$ for the fast and slow carriers, respectively. We note that the derivation of eq 1 assumes that one of the contacts is blocking and the other is Ohmic, which is clearly not a good assumption for the ambipolar carrier extraction that we observe. Thus, the mobilities that we extract using eq 1 are likely not highly accurate, but the order of magnitude difference in their values serves as a clear indicator that the main problem responsible for the $\mathrm{S}$ curve is misbalanced extraction of the two carriers.

To elucidate the reasons underlying this imbalanced charge extraction, we examined the structural characteristics of the BHJ/ cathode interface. Figure 3a shows an AFM phase image of the surface of the active layer of the P3HT:PCBM BHJ solar cell whose photo-CELIV transients are shown in Figure $2 \mathrm{~b}$. 

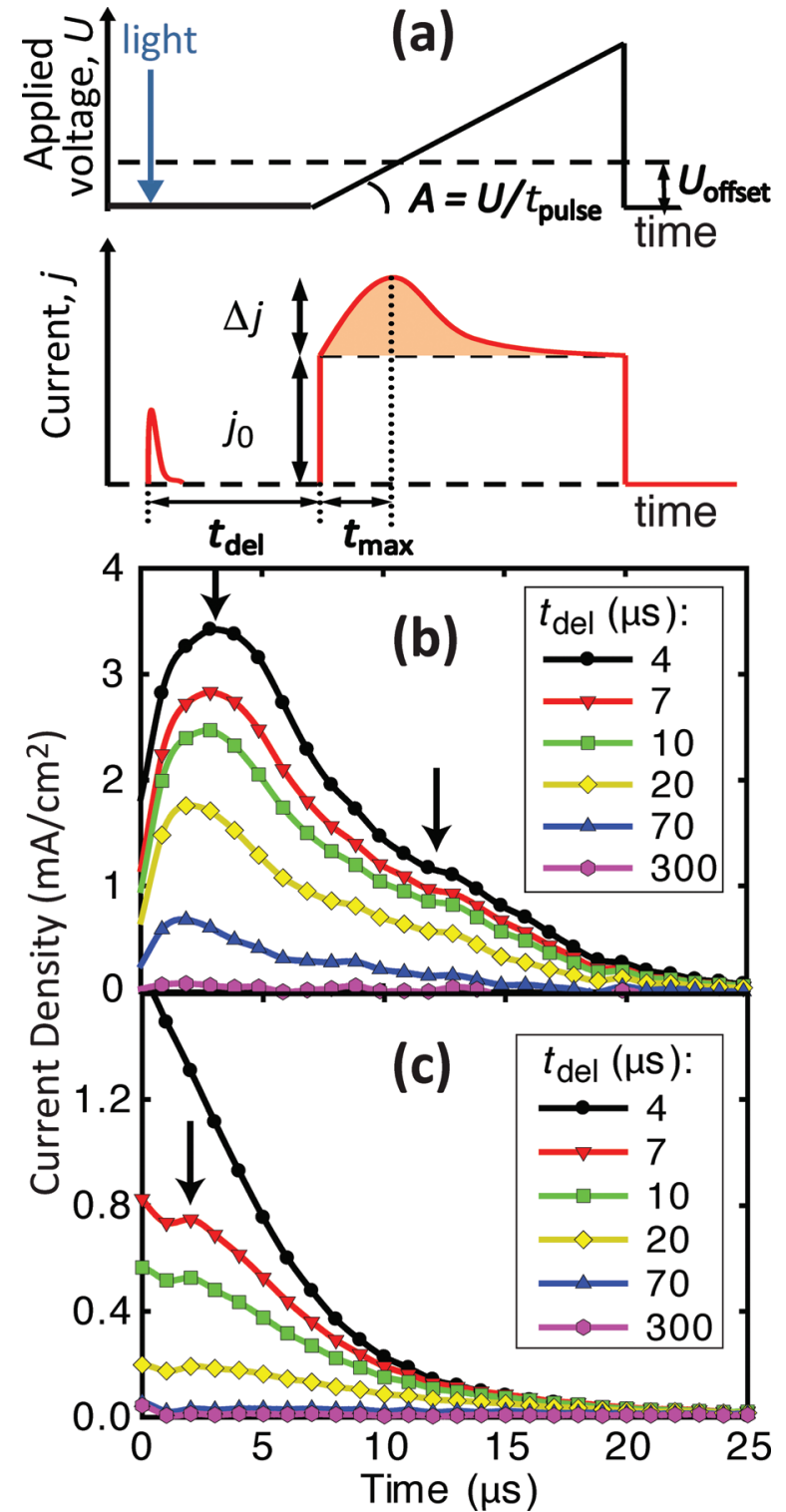

Figure 2. (a) Upper Panel: Timing schematic for the application of the light pulse and voltage ramp in photo-CELIV measurements. Lower Panel: Typical photo-CELIV current transient showing both capacitive $\left(j_{0}\right)$ and photoextracted currents (shaded area with amplitude $\Delta j$ ). (b) Photo-CELIV current transients, $j-j_{0}$, collected at different values of $t_{\text {del }}$ for the P3HT:PCBM BHJ device whose $J-V$ characteristics are given by the dashed curve in Figure 1. The two peaks indicated by the arrows correspond to the extraction of fast and slow carriers; we assign the fast peak to hole extraction and the slow peak to electron extraction for the reasons outlined in the text. (c) Photo-CELIV current transients, $j-j_{0}$, collected at different values of $t_{\text {del }}$ for a P3HT:PCBM device prepared at the same time as that in panel $b$, except that $\mathrm{a} \sim 20$ $\mathrm{nm}$ thick PCBM overlayer was spin-cast on top of the BHJ film before deposition of the cathode; the $J-V$ curve of this device is shown by the dot-dashed curve in Figure 1. The presence of a single peak indicates that electrons and holes are being extracted at roughly the same rate. The larger magnitude of the current transients and slower decay rate of the extracted current in panel $b$ relative to those in panel $\mathrm{c}$ are direct results of the improved electron extraction for the device in panel c, which causes more carriers to leak out of the active layer for a given value of $t_{\mathrm{del}}$. (See the Supporting Information for additional details.)

The image shows rice-like crystalline polymer domains with an average diameter of $11.6 \pm 2.7 \mathrm{~nm}$, and the film has a rms surface roughness of $0.68 \mathrm{~nm}$. These features are statistically identical to those obtained from the surface of pure P3HT films
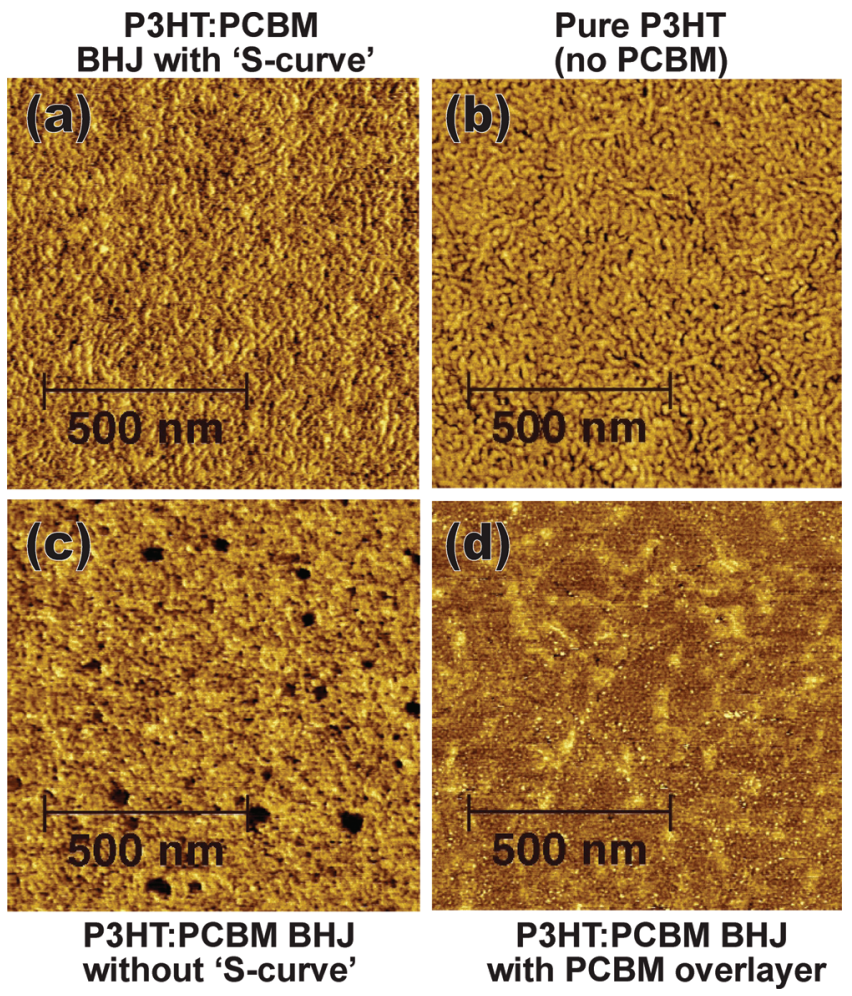

Figure 3. AFM tapping-mode phase images of the top surface of (a) the P3HT:PCBM BHJ film whose $S$ curve $J-V$ behavior is shown by the dashed curve in Figure 1 and whose photo-CELIV transients are shown in Figure $2 b$. The surface roughness of this film is $1.55 \mathrm{~nm}$, and the diameter of the P3HT nanocrystalline domains is $11.6 \pm 2.7$ $\mathrm{nm}$; (b) a pure P3HT film spun from ODCB, whose surface topography and phase image features are statistically identical to those of the BHJ film shown in panel a, indicating that the surface of the $\mathrm{S}$ curve BHJ film is nearly entirely composed of P3HT; (c) a P3HT:PCBM film whose $J-V$ characteristics show the "typical" behavior similar to the solid curve in Figure 1. The "rice-grain"-like structure seen in panels $a$ and $b$ is absent because the presence of PCBM near the top surface of the film interferes with P3HT's ability to form nanocrystalline domains. The large dark features (which correspond to bumps in the topography that give a rms surface roughness of $1.5 \mathrm{~nm}$ ) correspond to PCBM-rich regions at the surface of the film; (d) the P3HT:PCBM BHJ film with the $\sim 20 \mathrm{~nm}$ PCBM overlayer spin-cast from DCM, whose photo-CELIV transients are shown in Figure 2c and whose $J-V$ behavior is shown as the dot-dashed curve in Figure 1. The image shows a few $6.6 \mathrm{~nm}$ average diameter nanocrystallites of PCBM on an amorphous PCBM background, indicating that the mostly amorphous PCBM overlayer entirely covers the surface of the underlying BHJ.

cast from this same solvent, as shown in Figure 3b. It is striking that the surface of the $\mathrm{S}$ curve device shows none of the features assigned by others ${ }^{25}$ to PCBM-rich domains, which are visible on the surface of BHJ films that comprise the active layer of solar cells that do not exhibit the $\mathrm{S}$ curve behavior. We show an AFM image of the top surface of a BHJ film that gives typical device performance in Figure 3c; the rice-like grain structure of the polymer is suppressed since PCBM breaks up the nanoscale crystallinity of the P3HT. The dark features in this phase image, which correspond to bumps in topography, indicate that PCBM-rich domains are present at the top surface of the film, consistent with transmission electron microscopy work by others. ${ }^{26}$ The fact that the surfaces of BHJ films in good devices show evidence for PCBM at the top surface while BHJ films that show the $\mathrm{S}$ curve have a top surface that is nearly entirely composed of P3HT explains the poor performance of these devices; a good P3HT:PCBM solar cell needs PCBM on the surface to be in contact with the cathode for efficient extraction of electrons from the device. 
We believe that the lack of PCBM on the surface of BHJ films that give poor photovoltaic performance results from vertical phase segregation that takes place during spin-coating; this phase segregation drives PCBM toward the bottom of the film, away from the top surface in contact with the cathode. ${ }^{17,18}$ This type of behavior is expected for BHJ systems in which the PCBM component is much more soluble in the ODCB solvent than that in the P3HT polymer. ${ }^{27}$ During spin-coating, particularly under very dry conditions, the solvent evaporates first from the top of the film but is retained toward the substrate. This causes the PCBM component to segregate toward the solvent-rich (i.e., substrate) side of the BHJ film. In accordance with this idea, techniques such as solvent annealing ${ }^{4,28}$ or the addition of slowly evaporating solvent additives ${ }^{7}$ are known to modify the vertical distribution of solvent (and hence PCBM) in the film during the evaporation process and thus can greatly improve BHJ device performance, ${ }^{29}$ the presence of solvent vapor in the atmosphere during spin-coating can reduce the solvent gradient within the film during evaporation and thus reduce the amount of vertical phase separation. Unfortunately, the details of the solvent evaporation kinetics can be difficult to control experimentally, so that small changes in the degree of vertical phase separation of the polymer and fullerene components in the BHJ film lead to irreproducible device performance that, in extreme cases, results in the $\mathrm{S}$ curve. In the Supporting Information, we show that changing the drying conditions following spin-coating of our P3HT:PCBM BHJ active layers can have a large effect on the $\mathrm{S}$ curve; $\mathrm{BHJ}$ samples that were placed under vacuum to remove solvent from the film immediately after spin-coating are highly likely to show the S curve, whereas samples prepared under identical conditions that were allowed to dry in the presence of solvent vapor almost never show the $\mathrm{S}$ curve and have much more reproducible $J-V$ characteristics.

To further test our hypothesis that changes in the film's vertical composition profile due to subtleties in the spin-coating process are what is responsible for the $\mathrm{S}$ curve, we investigated whether or not we could improve both device performance and reproducibility by altering the film structure to increase the amount of PCBM at the top surface of the film in contact with the cathode. To do this, we simply spin-coated additional PCBM from a $0.5 \% \mathrm{w} / \mathrm{v}$ solution in dichloromethane (DCM) on top of the active BHJ layer at $4000 \mathrm{rpm}$ for $10 \mathrm{~s}$, producing a $\sim 20$ $\mathrm{nm}$ thick PCBM overlayer. We have shown elsewhere that the morphology of P3HT films cast from ODCB is not altered when a DCM solution is spun on top of the P3HT layer. ${ }^{30}$ In Figure $3 \mathrm{~d}$, we show an AFM phase image of a BHJ film onto which a PCBM overlayer has been spun from DCM; the surface shows all of the same features (occasional PCBM nanocrystals embedded in an amorphous PCBM background) as the PCBM overlayers that we spun onto pure P3HT films in our previous work ${ }^{30}$ Thus, the AFM image in Figure $3 \mathrm{~d}$ strongly suggests that the additional PCBM completely covers the surface of the BHJ film. On the basis of this result and the device data shown below, we believe that the act of spin-coating a PCBM overlayer onto a BHJ film accomplishes two things; it introduces additional PCBM onto the top surface of the BHJ film, and it leads to dissolution and redistribution of the PCBM within the BHJ, (partially) reversing some of the undesired vertical phase segregation. In addition, placing excess PCBM at the cathode interface may prevent possible chemical reactions between P3HT and the cathode, which have been suggested by others to cause poor device performance. ${ }^{11,13}$
The dot-dashed curve in Figure 1 shows the $J-V$ characteristics of the solar cell fabricated from the BHJ film with the PCBM overlayer whose AFM image is shown in Figure 3d; this device was made at the same time and under identical conditions as the device without the PCBM overlayer whose photo-CELIV transients are shown in Figure $2 \mathrm{~b}$. The figure makes it clear that the addition of the PCBM overlayer has "repaired" the S curve. We note that all of the BHJ devices with PCBM overlayers that we have tested, including those with BHJ thicknesses in the range of $80-115 \mathrm{~nm}$, show high fill factors; the addition of the PCBM overlayer not only eliminates the $\mathrm{S}$ curve, but it also creates devices whose performance characteristics are much more reproducible than those fabricated with the BHJ layer alone. Spinning PCBM overlayers from solutions with concentrations lower than $0.5 \% \mathrm{w} / \mathrm{v}$ also prevented the $\mathrm{S}$ curve, but the device performance was not as good as devices with overlayers spun from $0.5 \% \mathrm{w} / \mathrm{v}$ solutions.

We can see the reason that the PCBM overlayer improves device performance in Figure 2c, which shows photo-CELIV transients for the solar cell with the PCBM overlayer whose $J-V$ characteristics are shown as the dot-dashed curve in Figure 1 and whose AFM image is shown in Figure 3d. Figure $2 \mathrm{c}$ shows only a single peak in the extracted current at early times, $t_{\max }=1.8 \mu \mathrm{s}$, which via eq 1 corresponds to an effective carrier mobility of $\mu_{\text {avg }}=5 \times 10^{-4} \mathrm{~cm}^{2} \mathrm{~V}^{-1} \mathrm{~s}^{-1}$. The fact that we see only a single peak suggests that electrons and holes are being extracted at roughly the same rate from this device. The higher calculated effective mobility relative to the device in Figure $2 b$ indicates that we have improved the extraction of both types of carriers; the improvement in electron extraction prevents space charge buildup, which in turn improves the extraction of holes. Thus, we assign the fast and slow extraction peaks in Figure $2 b$ to the extraction of holes and electrons, respectively. Usually, photo-CELIV measurements cannot identify carrier types, but in this case, we can make the assignment because we know that electron extraction is specifically improved by inserting the additional PCBM layer between the BHJ and cathode, causing the longer-time exctraction peak to disappear. We note that the magnitude of the extracted charge in Figure 2c is less than that in Figure $2 b$ because the device with the PCBM overlayer has better contacts that allow a higher fraction of the photogenerated charges to leak out of the active layer during $t_{\text {del }}$, as discussed in more detail in the Supporting Information.

In summary, we have investigated the reasons that the performance of P3HT:PCBM BHJ solar cells fabricated using nominally identical conditions is irreproducible, which in the most extreme cases leads to poor devices that have an $\mathrm{S}$-shaped $J-V$ curve. Using the transient current technique photo-CELIV, we found that mismatched hole and electron charge carrier extraction rates are the cause of the reduced fill factor S curve. AFM images of the BHJ active layer in devices that exhibit the $\mathrm{S}$ curve showed that the composition of the top surface of the film is dominated by P3HT, the result of vertical phase segregation that is controlled by subtle factors in the solvent evaporation kinetics during spin-coating. When this type of vertical phase separation occurs, electron extraction is hindered by the poor contact between the PCBM component of the BHJ and the cathode. We were able to repair this poor contact by spin-coating an additional PCBM layer onto the BHJ film, reversing some of the undesired vertical phase segregation and improving electron collection at the cathode. Perhaps most importantly, unlike single-layer BHJ devices, the performance of BHJ solar cells with a 
PCBM overlayer are much more reproducible from batchto-batch and day-to-day, something that is a key requirement if polymer-based photovoltaics are to fulfill their promise in commercial applications.

Acknowledgment. This work was supported by the National Science Foundation under Grant Number CHE-0527015 and the Office of Naval Research under Contract Number N-0001404-1-0410. The authors thank Jordan Aguirre and Monica So for their help with sample preparation and testing.

Supporting Information Available: Details of the experimental techniques used in this work and the results of additional experiments that are mentioned briefly in the text. This material is available free of charge via the Internet at http://pubs.acs.org.

\section{References and Notes}

(1) Mayer, A. C.; Scully, S. R.; Hardin, B. E.; Rowell, M. W.; McGehee, M. D. Mater. Today 2007, 10, 28-33.

(2) Gunes, S.; Neugebauer, H.; Sariciftci, N. S. Chem. Rev. 2007, 107, 1324-1338.

(3) Park, S. H.; Roy, A.; Beaupre, S.; Cho, S.; Coates, N.; Moon, J. S.; Moses, D.; Leclerc, M.; Lee, K.; Heeger, A. J. Nat. Photon. 2009, 3, $297-$ 302 .

(4) Chen, L.-M.; Hong, Z.; Li, G.; Yang, Y. Adv. Mater. 2009, 21, 1434-1449.

(5) Hoppe, H.; Sariciftci, N. S. J. Mater. Chem. 2006, 16, 45-61.

(6) Reyes-Reyes, M.; Kim, K.; Carroll, D. L. Appl. Phys. Lett. 2005, 87, 083506-3.

(7) Peet, J.; Kim, J. Y.; Coates, N. E.; Ma, W. L.; Moses, D.; Heeger, A. J.; Bazan, G. C. Nat. Mater. 2007, 6, 497-500.

(8) Lee, J. K.; Ma, W. L.; Brabec, C. J.; Yuen, J.; Moon, J. S.; Kim, J. Y.; Lee, K.; Bazan, G. C.; Heeger, A. J. J. Am. Chem. Soc. 2008, 130, $3619-3623$.

(9) Ma, W. L.; Yang, C. Y.; Gong, X.; Lee, K.; Heeger, A. J. Adv. Funct. Mater. 2005, 15, 1617-1622.

(10) Reyes-Reyes, M.; Kim, K.; Dewald, J.; Lopez-Sandoval, R.; Avadhanula, A.; Curran, S.; Carroll, D. L. Org. Lett. 2005, 7, 5749-5752.

(11) Gupta, D.; Bag, M.; Narayan, K. S. Appl. Phys. Lett. 2008, 92 , 093301-3.
(12) Bisquert, J.; Garcia-Belmonte, G.; Munar, A.; Sessolo, M.; Soriano, A.; Bolink, H. J. Chem. Phys. Lett. 2008, 465, 57-62.

(13) Glatthaar, M.; Riede, M.; Keegan, N.; Sylvester-Hvid, K.; Zimmermann, B.; Niggemann, M.; Hinsch, A.; Gombert, A. Sol. Energy Mater. Sol. Cells 2007, 91, 390-393.

(14) Glatthaar, M.; Mingirulli, N.; Zimmermann, B.; Ziegler, T.; Kern, R.; Niggemann, M.; Hinsch, A.; Gombert, A. Phys. Status Solidi A 2005, 202, R125-R127.

(15) Jin, H.; Tuomikoski, M.; Hiltunen, J.; Kopola, P.; Maaninen, A.; Pino, F. J. Phys. Chem. C 2009, 113, 16807-16810.

(16) Juska, G.; Arlauskas, K.; Viliunas, M.; Genevicius, K.; Osterbacka, R.; Stubb, H. Phys. Rev. B 2000, 62, R16235.

(17) Campoy-Quiles, M.; Ferenczi, T.; Agostinelli, T.; Etchegoin, P. G.; Kim, Y.; Anthopoulos, T. D.; Stavrinou, P. N.; Bradley, D. D. C.; Nelson, J. Nat. Mater. 2008, 7, 158-164.

(18) Xu, Z.; Chen, L.-M.; Yang, G.; Huang, C.-H.; Hou, J.; Wu, Y.; Li, G.; Hsu, C.-S.; Yang, Y. Adv. Funct. Mater. 2009, 19, 1227-1234.

(19) Juska, G.; Arlauskas, K.; Viliunas, M.; Kocka, J. Phys. Rev. Lett. 2000, 84, 4946-4949.

(20) Mozer, A. J.; Dennler, G.; Sariciftci, N. S.; Westerling, M.; Pivrikas, A.; Osterbacka, R.; Juska, G. Phys. Rev. B 2005, 72, 035217.

(21) Mozer, A. J.; Sariciftci, N. S.; Pivrikas, A.; Osterbacka, R.; Juska, G.; Brassat, L.; Bassler, H. Phys. Rev. B 2005, 71, 035214.

(22) Dennler, G.; Mozer, A. J.; Juska, G.; Pivrikas, A.; Osterbacka, R.; Fuchsbauer, A.; Sariciftci, N. S. Org. Electron. 2006, 7, 229-234.

(23) Pivrikas, A.; Sariciftci, N. S.; Juska, G.; Osterbacka, R. Prog. Photovoltaics 2007, 15, 677-696.

(24) Juska, G.; Viliunas, M.; Arlauskas, K.; Nekrasas, N.; Wyrsch, N.; Feitknecht, L. J. Appl. Phys. 2001, 89, 4971-4974.

(25) Li, G.; Shrotriya, V.; Yao, Y.; Huang, J.; Yang, Y. J. Mater. Chem. 2007, 17, 3126-3140.

(26) Yang, X.; Loos, J.; Veenstra, S. C.; Verhees, W. J. H.; Wienk, M. M.; Kroon, J. M.; Michels, M. A. J.; Janssen, R. A. J. Nano Lett. 2005, $5,579-583$.

(27) Troshin, P. A.; Hoppe, H.; Renz, J.; Egginger, M.; Mayorova, J. Y.; Goryachev, A. E.; Peregudov, A. S.; Lyubovskaya, R. N.; Gobsch, G.; Sariciftci, N. S.; Razumov, V. F. Adv. Funct. Mater. 2009, 19, 779-788.

(28) Li, G.; Yao, Y.; Yang, H.; Shrotriya, V.; Yang, G.; Yang, Y. Adv. Funct. Mater. 2007, 17, 1636-1644.

(29) Dante, M.; Garcia, A.; Nguyen, T.-Q. J. Phys. Chem. C 2009, 113, $1596-1600$.

(30) Ayzner, A. L.; Tassone, C. J.; Tolbert, S. H.; Schwartz, B. J. J. Phys. Chem. C 2009, doi: 10.1021/jp9050897.

JP9082163 